06

\title{
Новые кластерные вторичные ионы для количественного анализа концентрации атомов бора в алмазе методом времяпролетной вторично-ионной масс-спектрометрии
}

\author{
(c) М.Н. Дроздов ${ }^{1}$, Ю.Н. Дроздов ${ }^{1}$, М.А. Лобаев ${ }^{2}$, П.А. Юнин ${ }^{1,3}$ \\ ${ }^{1}$ Институт фоииики микроструктур РАН, Нижний Новгород, Россия \\ ${ }^{2}$ Институт прикладной фризики РАН, Нижний Новгород, Россия \\ ${ }^{3}$ Нижегородский государственный университет им. Н.И. Лобачевского, \\ Нижний Новгород, Россия \\ E-mail: drm@ipm.sci-nnov.ru
}

Поступило в Редакцию 17 ноября 2017 г.

Предложен новый подход для количественного анализа концентрации атомов бора в алмазе на установках вторично-ионной масс-спектрометрии с времяпролетным масс-анализатором. Кроме известных линий масс-спектра, содержащих бор: $\mathrm{B}, \mathrm{BC}$ и $\mathrm{BC}_{2}$, обнаружено большое количество линий кластерных вторичных ионов $\mathrm{BC}_{N}$, их интенсивность на один-два порядка возрастает при использовании зондирующих ионов $\mathrm{Bi}_{3}$. Наибольшую интенсивность в порядке убывания имеют линии $\mathrm{BC}_{4}, \mathrm{BC}_{6}, \mathrm{BC}_{2}$ и $\mathrm{BC}_{8}$, при суммировании четырех наиболее сильных линий чувствительность возрастает на порядок в сравнении с известным режимом регистрации $\mathrm{BC}_{2}$. В монокристаллических пленках алмаза, выращенных в оптимальных условиях, измерены характеристики дельтаслоя бора с рекордными параметрами: ширина дельта-слоя порядка $2 \mathrm{~nm}$, концентрация $6.4 \cdot 10^{20} \mathrm{~cm}^{-3} \mathrm{c}$ перепадом на четыре порядка по концентрации бора для легированного и нелегированного алмаза.

DOI: 10.21883/PJTF.2018.07.45885.17121

Основные принципы количественного анализа концентрации атомов примеси в полупроводниках $\mathrm{Si}$, GaAs и алмазе методом вторичноионной масс-спектрометрии (ВИМС) были разработаны более 20 лет назад [1]. Для регистрации элемента примеси $E$ используются вторичные ионы с максимальной интенсивностью (элементарные $E^{+}$ 
или $E^{-}$при распылении ионами кислорода или цезия соответственно) или кластерные $\left(E M^{-}, E \mathrm{Cs}^{+}\right.$при распылении ионами цезия, $M-$ элемент матрицы). Этот подход с небольшими изменениями сохранился до сих пор. В работе [2] отмечается, что для структур алмаза кроме $E, E C^{-}$могут использоваться и кластерные вторичные ионы $E \mathrm{C}_{2}^{-}$ c сопоставимой интенсивностью. Методика количественного анализа концентрации атомов примеси в структурах алмаза оказалась очень востребованной в последнее время для послойного анализа монокристаллических пленок алмаза с дельта-слоями бора. Такие структуры рассматриваются как новый перспективный материал для современной микроэлектроники [3-7]. Для послойного анализа дельта-слоев требуется сочетание нескольких факторов: высокого разрешения по глубине, высокой элементной чувствительности и большого динамического диапазона регистрируемой концентрации, что не всегда достигается в экспериментах. Решению этих вопросов посвящено большое число работ [8-11]. Отметим, что указанные методики количественного анализа концентрации атомов примеси $[1,2]$ были разработаны для установок ВИМС с магнитосекторным масс-анализатором фирмы Сатеса. Нам неизвестны подобные систематические исследования для установок ВИМС другого типа - с времяпролетным масс-анализатором TOF.SIMS-4 (5). Между тем эти установки в последнее время находят все большее распространение как наиболее универсальный инструмент, сочетающий возможности статического анализа молекулярного состава поверхности и динамического послойного анализа атомного состава полупроводниковых структур. В настоящей работе изучаются новые возможности и подходы для количественного анализа концентрации атомов бора в алмазе методом времяпролетной вторично-ионной массспектрометрии на установке TOF.SIMS-5. Прежде всего обратим внимание на особенности формирования масс-спектров вторичных ионов в углеродсодержащих материалах. В работах $[12,13]$ было показано, что в масс-спектрах вторичных ионов структур на основе углерода, таких как карбид бора или фуллерены, присутствует очень большое число кластерных линий углерода $\mathrm{C}_{N}(N=2-20$ и выше) и их комбинаций с другими атомами. Это очевидно связано со спецификой химической связи атомов углерода, образующих основу всех органических соединений на Земле. Кластерные линии $C_{N}$ регистрируются не только в статическом режиме ВИМС, но и при послойном анализе структур с распылением ионами цезия. Интенсивность таких линий с $N>3$

Письма в ЖТФ, 2018, том 44, вып. 7 
резко возрастает при использовании для зондирования кластерных ионов $\mathrm{Bi}_{3}$ вместо элементарных ионов Вi. Причина этого возрастания связана с изменением характера развития каскада столкновений: при зондировании поверхности элементарными ионами Ві доминируют бинарные столкновения, в случае $\mathrm{Bi}_{3}$ развивается режим тепловых пиков, при котором в каскаде устанавливается высокая эффективная температура и резко возрастает эмиссия молекулярных и многоатомных кластерных вторичных ионов [14]. Поэтому можно ожидать, что при количественном анализе структур алмаза, легированных бором, набор регистрируемых кластерных вторичных ионов может быть значительно шире, чем это известно из литературы и используется до сих пор. Такая возможность представляет практический интерес, поскольку может повысить элементную чувствительность и расширить динамический диапазон регистрации атомов бора.

В работе изучались монокристаллические пленки алмаза с дельтаслоями бора, выращенные на монокристаллических подложках фирмы New Diamond Technology. Рост пленок проводился на разработанном в ИПФ РАН CVD-реакторе, в котором в газовой смеси $\mathrm{H}_{2}-\mathrm{CH}_{4}$ излучением магнетрона поддерживалась плазма СВЧ-разряда. Для роста дельта-слоев газовая смесь кратковременно заменялась на газовую смесь с дибораном $\mathrm{H}_{2}-\mathrm{CH}_{4}-\mathrm{B}_{2} \mathrm{H}_{6}[6,7]$. В качестве геттера бора использовалась сера, которая связывает атомы бора и уменьшает их вхождение в растущую пленку, что позволяет повысить резкость переходов дельта-слоев [7,15]. Измерения методом ВИМС выполнялись на установке TOF.SIMS-5 с раздельными функциями ионных пучков распыление-зондирование. Распыление проводилось ионами $\mathrm{Cs}^{+} \mathrm{c}$ энергией $1 \mathrm{keV}$, зондирование - ионами $\mathrm{Bi}^{+}$или $\mathrm{Bi}_{3}^{+} \mathrm{c}$ энергией $25 \mathrm{keV}$. Регистрировались отрицательные вторичные ионы. Количественная калибровка чувствительности к атомам бора проводилась с использованием тестовой структуры $A-$ монокристаллического алмаза, имплантированного изотопом ${ }^{11}$ В. Изучались структуры $B$ и $C$ с дельтаслоями бора, различавшиеся режимом роста.

На рис. 1 показаны интенсивности линий масс-спектра вторичных ионов, содержащих бор: $\mathrm{BC}_{N-1}(N=1-11)$ - для монокристаллической пленки алмаза с однородным легированием бором с концентрацией $5 \cdot 10^{19} \mathrm{~cm}^{-3}$. При зондировании ионами Ві наиболее сильными линиями в масс-спектре являются $\mathrm{BC}_{2}$ и $\mathrm{BC}_{4}$, их интенсивности близки. При зондировании ионами $\mathrm{Bi}_{3}$ интенсивность кластерных вторичных ионов

Письма в ЖТФ, 2018, том 44, вып. 7 


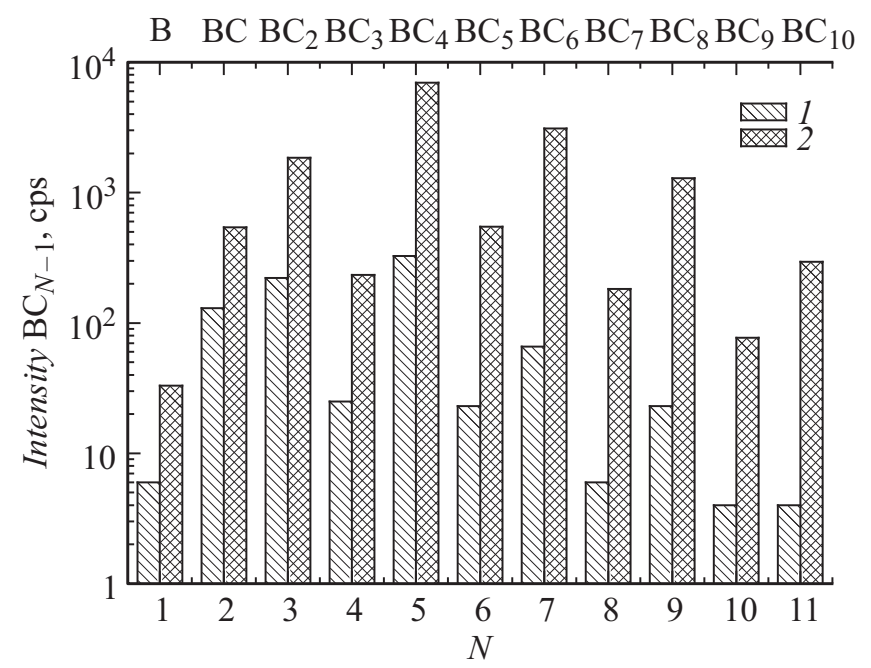

Рис. 1. Интенсивности линий $\mathrm{BC}_{N-1}(N=1-11)$ масс-спектра вторичных ионов, содержащих бор, для монокристаллической пленки алмаза с однородным легированием бором. 1 - для зондирующих ионов $\mathrm{Bi}, 2$ - для зондирующих ионов $\mathrm{Bi}_{3}$.

$\mathrm{BC}_{N}$ существенно возрастает, для наиболее тяжелых вторичных ионов это различие составило около 100 раз. При этом максимум интенсивностей линий смещен в сторону больших значений $N$ : интенсивности $\mathrm{BC}_{4}$ и $\mathrm{BC}_{6}$ превышают интенсивность $\mathrm{BC}_{2}$ соответственно в 4 и 1.5 раза. Интенсивность линии $\mathrm{BC}_{8}$ сопоставима с $\mathrm{BC}_{2}$. Наибольшую интенсивность имеют линии с нечетным значением $N$. Поэтому оптимальным режимом количественного анализа концентрации атомов бора в алмазе оказывается использование зондирующих ионов $\mathrm{Bi}_{3}$ и суммирование четырех линий масс-спектра: $\mathrm{BC}_{2}, \mathrm{BC}_{4}, \mathrm{BC}_{6}$ и $\mathrm{BC}_{8}$. Суммарный вклад остальных линий масс-спектра с ${ }^{11} \mathrm{~B}$ не превышает 5-7\%. При необходимости суммарная интенсивность может быть повышена еще на $\sim 20 \%$ за счет использования линий с изотопом ${ }^{10} \mathrm{~B}$.

На рис. 2 приведен профиль концентрации бора в тестовой структуре $A$, использованной для количественной калибровки чувствительности. Рис. 2 позволил определить чувствительность в оптимальном 


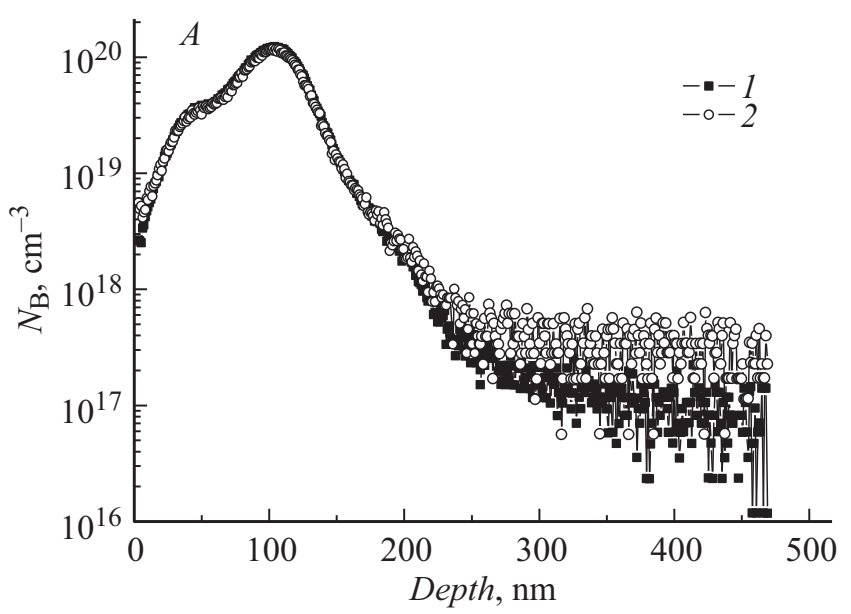

Рис. 2. Профили концентрации бора в тестовом образце $A$, использованном для количественной калибровки чувствительности. 1 - для суммы вторичных ионов, расчет по выражению (1), 2 - для вторичных ионов $\mathrm{BC}_{2}$, расчет по выражению (2).

режиме калибровки

$$
N_{\mathrm{B}}=1.9 \cdot 10^{21}\left\{I\left(\mathrm{BC}_{2}\right)+I\left(\mathrm{BC}_{4}\right)+I\left(\mathrm{BC}_{6}\right)+I\left(\mathrm{BC}_{8}\right)\right\} / I\left(\mathrm{C}_{8}\right),
$$

где $I\left(\mathrm{BC}_{N}\right), I\left(\mathrm{C}_{8}\right)$ - интенсивности вторичных ионов. Нормировка на элемент матрицы проводится на кластерные вторичные ионы $\mathrm{C}_{8}$, поскольку интенсивность кластерных линий с меньшим числом атомов углерода оказывается насыщенной из-за высокого значения. С использованием только линии $\mathrm{BC}_{2}$ выражение (1) принимает вид

$$
N_{\mathrm{B}}=2 \cdot 10^{22} I\left(\mathrm{BC}_{2}\right) / I\left(\mathrm{C}_{8}\right) \text {. }
$$

В соответствии с данными работ $[1,2]$ численный множитель в выражениях (1) и (2) имеет значение фактора относительной чувствительности RSF. Для выражения (2) RSF на порядок превышает значение RSF для формулы (1). Поэтому элементная чувствительность в режиме (1) на порядок выше, чем для (2).

Письма в ЖТФ, 2018, том 44, вып. 7 
Рис. 2 также показывает разную величину фонового шумового сигнала $N_{\mathrm{B}}$, определяющего минимальный предел обнаружения бора в алмазе. Для (1) он составляет $(5-7) \cdot 10^{16} \mathrm{~cm}^{-3}$, для (2) $(3-5) \cdot 10^{17} \mathrm{~cm}^{-3}$. При вычитании интенсивности шумовых сигналов, определенных в соседних интервалах масс, реальный предел обнаружения бора снижается: для (1) он составляет $(1.2-1.4) \cdot 10^{16} \mathrm{~cm}^{-3}$, для (2) $-(6-7) \cdot 10^{16} \mathrm{~cm}^{-3}$. Эти значения были получены при измерении на нелегированных пленках алмаза. Таким образом, использование режима (1) дает заметный выигрыш в минимально регистрируемой концентрации атомов бора и динамическом диапазоне $N_{\mathrm{B}}$. Отметим, что уровень концентрации $10^{16} \mathrm{~cm}^{-3}$ является практически предельным при послойном анализе на установке TOF.SIMS-5. Однако он может быть снижен в 5-10 раз при использовании аналога статического режима ВИМС путем длительного времени интегрирования масс-спектра при низкой энергии распыляющих ионов.

На рис. 3 приведены профили $N_{\text {B }}$ в структурах алмаза $B$ и $C$ с дельта-слоями бора. В структуре $B$ значение $N_{\mathrm{B}}$ в максимуме дельтаслоя составляет $6.4 \cdot 10^{20} \mathrm{~cm}^{-3}$, ширина дельта-слоя на полувысоте $2.1 \mathrm{~nm}$. Насколько нам известно, эти параметры дельта-слоя являются рекордными в настоящее время. Эти значения получены непосредственно из эксперимента, обработка профиля с учетом функции разрешения по глубине может существенно их улучшить. Высокая концентрация в максимуме позволяет рассчитывать на металлический тип проводимости, а малая ширина - на уход значительной части свободных носителей (дырок) на более высокие энергетические уровни в потенциальной яме дельта-слоя, пространственно разделенные с легирующими атомами [3]. Это может позволить снизить примесное рассеяние и увеличить подвижность дырок. Характерная длина изменения концентрации на порядок величины $L_{D e c}$ составляет $1.1 \mathrm{~nm}$ для переднего фронта дельта-слоя и $2.1 \mathrm{~nm}$ для заднего. Затягивание заднего фронта связано с процессами атомного перемешивания при распылении ионами цезия $[7,8]$.

На рис. 3, $a$ результаты измерения основных параметров дельтаслоя, полученные по выражениям (1) и (2), практически совпадают. Разница возникает в определении параметров фонового уровня $N_{\mathrm{B}}$ в нелегированной части структуры, как отмечалось выше. В то же время при анализе дельта-слоев с меньшей концентрацией атомов бора заметная разница в результатах, полученных по формулам (1) и (2),

Письма в ЖТФ, 2018, том 44, вып. 7 

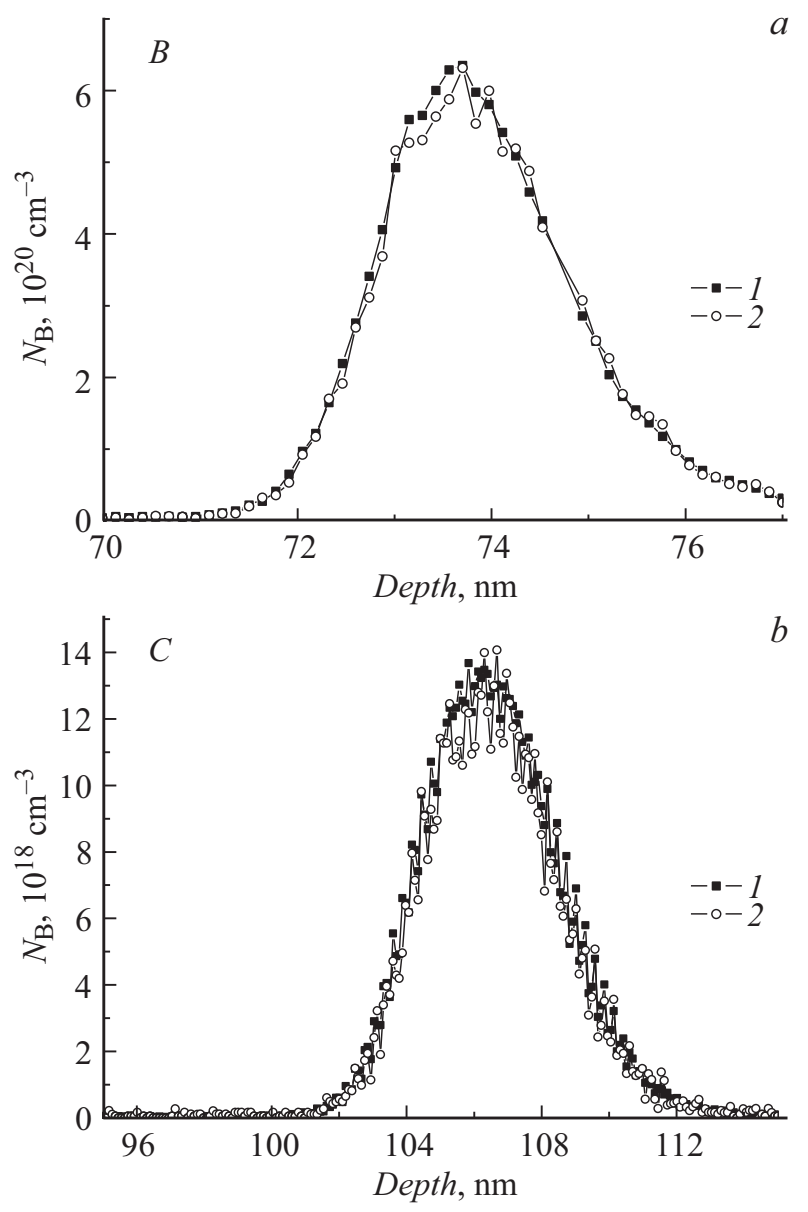

Рис. 3. Профили концентрации бора в структурах $B(a)$ и $C(b)$ с дельта-слоем бора. 1 - для суммы вторичных ионов, расчет по выражению (1), 2 - для вторичных ионов $\mathrm{BC}_{2}$, расчет по выражению (2).

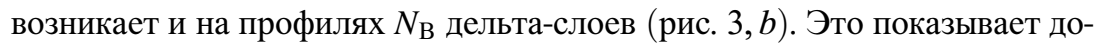
полнительное преимущество режима (1). Такое же преимущество будет возникать при анализе дельта-слоев с большей скоростью распыления, необходимой для анализа на бо́льшую глубину.

Письма в ЖТФ, 2018, том 44, вып. 7 
Таким образом, в работе впервые установлены новые возможности количественного анализа концентрации атомов бора в алмазе на установках с времяпролетным масс-анализатором. Показано, что кроме известных линий масс-спектра $\mathrm{B}, \mathrm{BC}$ и $\mathrm{BC}_{2}$ в масс-спектрах регистрируется большое количество линий кластерных вторичных ионов $\mathrm{BC}_{N}$, их интенсивность существенно возрастает при использовании зондирующих ионов $\mathrm{Bi}_{3}$. Наибольшую интенсивность в порядке убывания имеют линии $\mathrm{BC}_{4}, \mathrm{BC}_{6}, \mathrm{BC}_{2}$ и $\mathrm{BC}_{8}$. Определены факторы относительной чувствительности бора в алмазе для установки TOF.SIMS-5, при суммировании четырех наиболее сильных линий чувствительность возрастает на порядок в сравнении с известным режимом регистрации $\mathrm{BC}_{2}$. Выращены монокристаллические пленки алмаза с дельта-слоями бора с рекордными параметрами: ширина дельта-слоя порядка $2 \mathrm{~nm}$, концентрация в максимуме $6.4 \cdot 10^{20} \mathrm{~cm}^{-3}$ с перепадом на четыре порядка по концентрации бора для легированного и нелегированного алмаза. Реализованные возможности метода ВИМС позволят более детально изучить процесс роста дельта-слоев бора, в частности влияние подачи геттера бора-серы. По этому вопросу в литературе до сих пор нет единого мнения, поскольку сера может как связывать атомы бора и уменьшать их встраивание в алмаз, так и приводить к возникновению комплексов $\mathrm{S}-\mathrm{B}$, влияющих на тип проводимости.

Работа поддерживалась грантом Российского научного фонда (проект № 17-19-01580). В работе использовано оборудование ЦКП ИФМ РАН.

\section{Список литературы}

[1] Wilson R.G. // Int. J. Mass Spectrometry Ion Process. 1995. V. 143. P 43-49.

[2] Wilson R.G., Zavada J.M. // Mater. Sci. Eng. R. 2012. V. 73. P. 101-128.

[3] Chicot G., Fiori A., Volpe P.N., Tran Thi T.N., Gerbedoen J.C., Bousquet J., Alegre M.P., Pinero J.C., Araujo D., Jomard F., Soltani A., De Jaeger J.C., Morse J., Hartwig J., Tranchant N., Mer-Calfati C., Arnault J.C., Delahaye J., Grenet T., Eon D., Omnés F., Pernot J., Bustarret E. // J. Appl. Phys. 2014. V. 116. P. 083702.

[4] Donato N., Pagnano D., Napoli E., Longobardi G., Udrea F. // Diamond Rel. Mater. 2017. V. 78. P. 73-82.

[5] Fiori A., Jomard F., Teraji T., Koizumi S., Isoya J., Gheeraert E., Bustarret E. // Appl. Phys. Express. 2013. V. 6. P. 045801.

Письма в ЖТФ, 2018, том 44, вып. 7 
[6] Vikharev A.L., Gorbachev A.M., Lobaev M.A., Muchnikov A.B., Radishev D.B., Isaev V.A., Chernov V.V., Bogdanov S.A., Drozdov M.N., Butler J.E. // Phys. Status Solidi RRL. 2016. V. 10. P. 324-327.

[7] Butler J.E., Vikharev A., Gorbachev A., Lobaev M., Muchnikov A., Radischev D., Isaev V., Chernov V., Bogdanov S., Drozdov M., Demidov E., Surovegina E., Shashkin V., Davidov A., Tan H., Meshi L., Pakpour-Tabrizi A.C., Hicks M.-L., Jackman R.B. // Phys. Status Solidi RRL. 2017. V. 11. P. 1600329. DOI 10.1002/pssr.201600329

[8] Fiori A., Jomard F., Teraji T., Chicot G., Bustarret E. // Thin Solid Films. 2014. V. 557. P. 222-226.

[9] Guzmán de la Mata B., Sanz-Hervás A., Dowsett M.G., Schwitters M., Twitchen D. // Diamond Rel. Mater. 2007. V. 16. P. 809-814.

[10] Mer-Calfati C., Tranchant N., Volpe P.N., Jomard F., Weber S., Bergonzo P., Arnault J.C. // Mater. Lett. 2014. V. 115. P. 283-286.

[11] Ogura M., Kato H., Makino T., Okushi H., Yamasaki S. // J. Crystal Growth. 2011. V. 317. P. 60-63.

[12] Дроздов М.Н., Дроздов Ю.Н., Пахомов Г.Л., Травкин В.В., Юнин П.А. // Письма в ЖТФ. 2013. Т. 39. В. 24. С. 45-54.

[13] Ber B., Babor P., Brunkov P.N., Chapon P., Drozdov M.N., Duda R., Kazantsev D., Polkovnikov V.N., Yunin P., Tolstogouzov A. // Thin Solid Films. 2013. V. 540. P. 96-105.

[14] Van Vaeck L., Adriaens A., Gijbels R. // Mass Spectrometry Rev. 1999. V. 18. P. 1-47.

[15] Eaton S.C., Anderson A.B., Angus J.C., Evstefeeva Y.E., Pleskov Y.V. // Electrochem. Solid-State Lett. 2002. V. 5. N 8. P. G65-G68. 